\title{
Perceived overweight and suicidality among US adolescents from 1999 to 2017
}

\author{
Michael Daly ${ }^{1}$ Eric Robinson ${ }^{2} \cdot$ Angelina R. Sutin $^{3}$
}

Received: 27 January 2020 / Revised: 6 May 2020 / Accepted: 22 May 2020 / Published online: 16 June 2020

(c) Springer Nature Limited 2020

\begin{abstract}
Identifying oneself as overweight is a risk factor for poor mental health and suicidality independent from objective weight status. The stigma associated with heavier body weight has risen in recent decades and this may have exacerbated the detrimental mental health effects of perceived overweight. In this study, we examined the association between perceived overweight and suicidality in a nationally representative sample $(N=115,180)$ of US adolescents assessed from 1999 to 2017. We drew on data from the Youth Risk Behavior survey, a biennial population-based survey of students in grades 9-12. Suicidality was gauged by participant reports of past-year suicidal ideation, suicide plans, or suicide attempts. Across all waves, perceived overweight (vs. perceived "normal" weight) predicted a 7.7 percentage point $(p<0.001)$ increased risk of suicidality after adjustment for age, sex, ethnicity, and BMI. The risk of suicidality associated with perceived overweight increased from 5.7 percentage points in 1999-2001 to 10.1 points in 2015-2017, a difference of 4.4 points $(p=0.001)$. This growth was most evident after 2009 and was apparent across suicidality measures. Among US adolescents, perceiving one's body as overweight increases risk of suicidality and this risk appears to have grown substantially from 2009 to 2017 .
\end{abstract}

\section{Introduction}

Body mass index (BMI) is an inconsistent and complex predictor of suicidality [1-3]. For instance, while higher weight individuals are at increased risk of suicidal ideation, they are less likely to die by suicide [2]. In this study, we contend that the role of weight in predicting suicidality may depend on the psychological experience attached to heavier body weight [3]. Heavier body weight is widely stigmatized and experiencing discrimination based on weight is asso-

Supplementary information The online version of this article (https:// doi.org/10.1038/s41366-020-0620-9) contains supplementary material, which is available to authorized users.

Michael Daly

Michael.A.Daly@mu.ie

1 Department of Psychology, Maynooth University, Maynooth, Ireland

2 Department of Psychological Sciences, Institute of Psychology, Health \& Society, University of Liverpool, Liverpool, UK

3 Department of Behavioral Sciences and Social Medicine, College of Medicine, Florida State University, Tallahassee, FL, USA ciated with detrimental mental health effects $[4,5]$. As such, people who identify as overweight recognize that they possess a characteristic that is stigmatized and may anticipate being devalued or denigrated because of their weight. A recent meta-analysis [3] of observational evidence found that personal identification of overweight explained why BMI predicted increased suicidality. In addition, this study found that children and adolescents who identified as overweight were at an elevated risk of suicidality [3].

This finding is particularly concerning because largescale screening and feedback programs which involve notifying individuals of their weight status have been rolled out in schools in several countries including the USA and UK $[6,7]$. Further, there is evidence that the stigma associated with heavier body weight may have risen in recent decades. For instance, a national survey in the USA showed that self-reported experiences of discrimination based on body weight increased from 1995-1996 to 2004-2006 [8]. Another study showed an increase in explicit antifat bias amongst obesity specialists from 2001 to 2013 [9]. Further, from 2002 to 2014 , the extent to which perceived overweight was associated with relatively minor physical health complaints (e.g., headache, feeling low, feeling dizzy) increased in a sizeable proportion of European countries 
[10]. However, adverse mental health consequences were not examined.

Based on the increasing stigma associated with heavier body weight, we hypothesize that adolescents who selfidentify as overweight may be becoming increasingly vulnerable to the mental health consequences associated with self-perceived overweight, such as suicidality. In the present study we test this hypothesis using data from a large nationally representative sample of US adolescents.

\section{Methods}

\section{Participants}

Data were drawn from biennial waves of the Youth Risk Behavior Surveillance System (YRBSS) survey from 1999 to $2017(N=115,180)$ survey waves where perceived weight, suicidality, and participant BMI were assessed. The YRBSS is a nationally representative sample of high school students in grades 9-12 in the USA and the sampling strategy is detailed elsewhere [11]. Participants included in the present analyses were $50.1 \%$ female, and $62.3 \%$ White, $12 \%$ Black, $16.5 \%$ Hispanic, and $9.2 \%$ other races.

\section{Perceived weight}

Self-perceived weight was assessed using the item "How do you describe your weight" rated on a scale from 1 (very underweight) to 5 (very overweight), with a mid-point of 3 (about the right weight). Those classified as having the selfperception of overweight indicated they perceive their weight as slightly or very overweight. This group was compared with those who viewed their weight as "about the right weight" and were classified as perceiving their weight as "normal" for brevity and in line the existing literature on this topic [3]. Those who considered their weight to be slightly or very underweight were categorized as having the self-perception of underweight.

\section{Suicidality}

As a part of the YRBSS survey participants were asked about three forms of suicidality. First, suicidal ideation was measured by the survey question, "During the past 12 months, did you ever seriously consider attempting suicide?". Suicide plans were measured by the question, "During the past 12 months, did you make a plan about how you would attempt suicide?". Response options for both questions were "yes" or "no". Suicide attempt was assessed by the question, "During the past 12 months, how many times did you actually attempt suicide?" which was recoded to "0 times" = "no" and "1 time" or more = "yes". Those who responded "yes" to any of the three questions were coded as experiencing suicidality in the past year.

\section{Covariates}

Participants reported their age, sex, and race/ethnicity (White/ Black/Hispanic/other race) and their height and weight, which were used to determine their BMI. Depression was captured by "yes" responses to the item "During the past 12 months, did you ever feel so sad or hopeless almost every day for 2 weeks or more in a row that you stopped doing some usual activities?"

\section{Statistical analysis}

First, we specified a logistic regression model to test whether suicidality was associated with perceived overweight (vs. perceived normal weight) across all waves (1999-2017). We also included controls for participant age, sex, race/ethnicity, BMI, and survey period. To include all participants with available data in our analyses we also estimated the relationship between perceived underweight and suicidality as a part of our analyses. This relationship was outside the scope of the current paper and for this reason we include the relevant results in the Supplementary information (Table S2). To reduce the number of statistical comparisons made, in our main analyses we examined a single suicidality indicator (reporting either suicidal ideation, suicide plans, or suicide attempts in the past year) and grouped survey waves into five categories: 1999-2001/ 2003-2005/2007-2009/2011-2013/2015-2017.

To estimate changes in the association between perceived overweight and suicidality over time we added an interaction between survey period categories and perceived overweight to the model and estimated the probability of suicidality at each time period using the Stata margins command [12].

We then employed a difference-in-difference approach to estimate whether the average difference in suicidality between the perceived overweight and perceived normal weight groups changed after 1999-2001. In supplementary analyses we tested whether the study results were affected by adjusting for a categorical measure of body weight (underweight/normal weight/overweight/obesity) rather than the continuous BMI measure included in the main analyses. We conducted this additional analysis because prior research suggests that there may be a nonlinear relationship between BMI and suicidal ideation with those with low and high BMI levels at increased risk [13]. In addition, we tested whether adding the depression indicator described above to the main study models markedly changed the relationship between perceived overweight and suicidality. We did not include depression in the main study models as the feelings of sadness and hopelessness captured by 

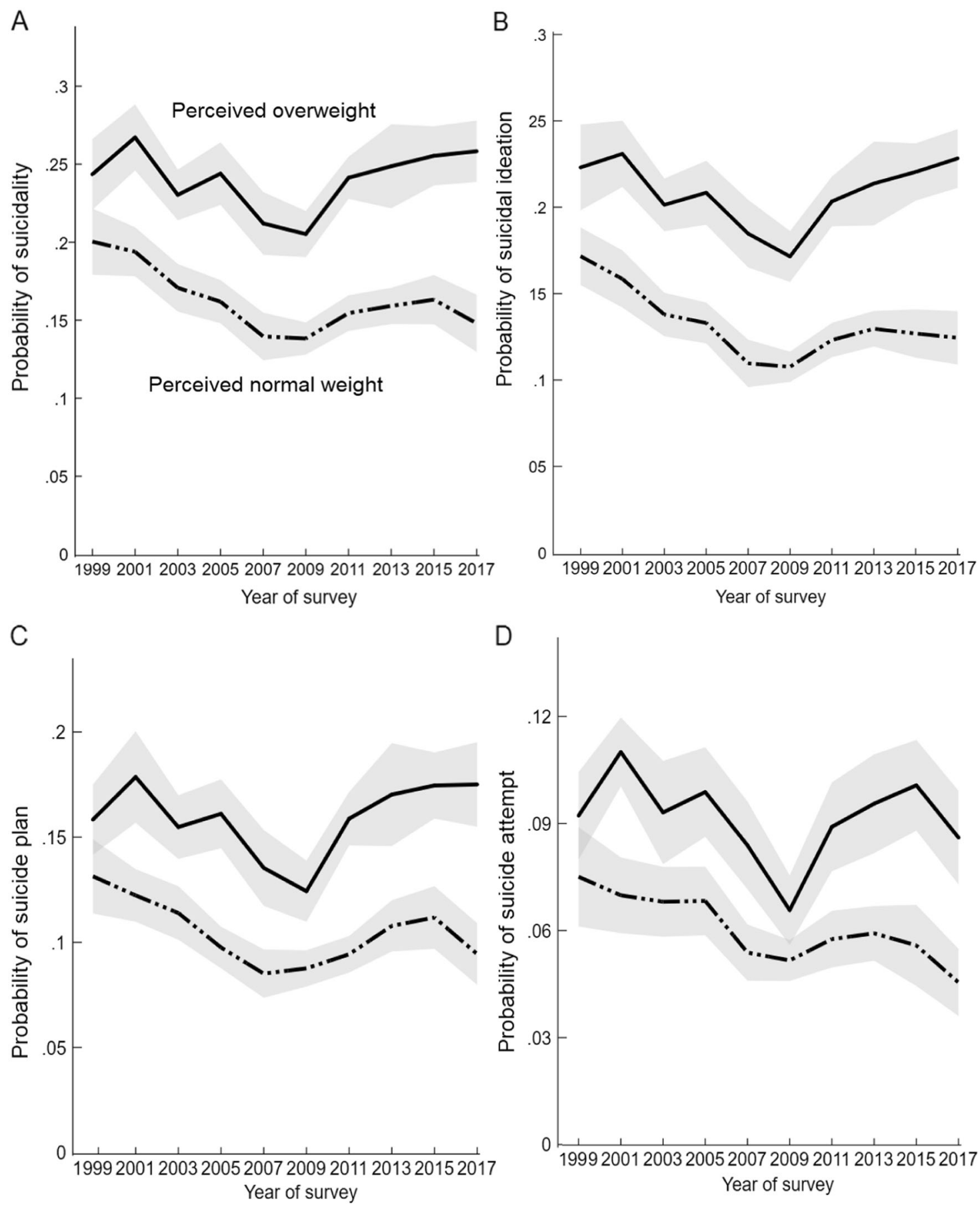

Fig. 1 Perceived overweight (vs. perceived normal weight) and suicide outcomes from 1999-2017. Predicted probability of participants with perceived overweight (solid line) and perceived normal weight (broken line) reporting suicidality (a), suicidal ideation (b),

this variable may be a potential pathway through which perceived overweight relates to suicidality. Finally, we estimated models separately for each of the three suicidality measures. All analyses were weighted to account for the sampling design (stratification, clustering, and unequal selection probabilities) [11]. suicide plans (c), and suicide attempts (d) at each wave of the Youth Risk Behavior Surveillance System (YRBSS) survey $(N=115,180)$. Estimates are adjusted for participant age, sex, race/ethnicity, and BMI. 95\% confidence intervals presented in gray.

\section{Results}

Descriptives by survey period are shown in Table S1. Across all waves, $30.3 \%$ of participants self-identified as overweight, whilst $55.3 \%$ identified as normal weight, and $14.4 \%$ identified as underweight. The prevalence of 
Table 1 Relationship between perceived overweight (vs. perceived normal weight) and the predicted probability of suicidality from 1999 to $2017(\mathrm{~N}=115,180)$.

\begin{tabular}{|c|c|c|}
\hline & $\begin{array}{l}\text { Probability of suicidality } \\
(95 \% \text { CI })\end{array}$ & $p$ \\
\hline \multicolumn{3}{|c|}{ Perceived overweight in } \\
\hline 1999-2001 & $0.057(0.036,0.078)$ & $<0.001$ \\
\hline $2003-2005$ & $0.070(0.053,0.087)$ & $<0.001$ \\
\hline 2007-2009 & $0.070(0.052,0.087)$ & $<0.001$ \\
\hline 2011-2013 & $0.089(0.072,0.105)$ & $<0.001$ \\
\hline $2015-2017$ & $0.101(0.086,0.116)$ & $<0.001$ \\
\hline \multicolumn{3}{|c|}{ Perceived overweight in } \\
\hline $\begin{array}{l}2003-2005 \text { vs. } \\
1999-2001^{\text {b }}\end{array}$ & $0.013(-0.013,0.039)$ & 0.324 \\
\hline $\begin{array}{l}2007-2009 \text { vs. } \\
1999-2001^{\text {b }}\end{array}$ & $0.013(-0.013,0.039)$ & 0.339 \\
\hline $\begin{array}{l}2011-2013 \text { vs. } \\
1999-2001^{\text {b }}\end{array}$ & $0.032(0.006,0.057)$ & 0.016 \\
\hline $\begin{array}{l}2015-2017 \text { vs. } \\
1999-2001^{\text {b }}\end{array}$ & $0.044(0.019,0.069)$ & 0.001 \\
\hline
\end{tabular}

Models are adjusted for participant age, gender, race/ethnicity, BMI, and include a dummy variable identifying those who perceive themselves as underweight.

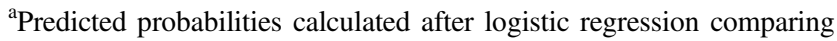
perceived overweight to perceived normal weight.

${ }^{b}$ Models compare the difference in the probability of suicidality between perceived overweight and perceived normal weight groups in 1999-2001 with the differences identified in subsequent survey periods. Difference-in-difference coefficients were calculated using the "lincom" command in Stata.

perceived overweight was lowest in 2007-2009 (28.3\%) and highest in 2015-2017 (31.9\%). 19.8\% of participants reported experiencing suicidality in the past year. On average, those who perceived themselves to be overweight (vs. perceived normal weight) experienced an increased predicted probability of suicidality of 7.7 percentage points (95\% CI $(6.79-8.55, p<0.001)$ ), after adjustment for demographic characteristics and BMI.

An initial trend analysis suggested that the predicted probability of suicidality associated with perceived overweight was relatively stable from 1999-2009 and increased subsequently (see Fig. 1). The predicted probabilities of suicidality associated with perceived overweight are summarized in Table 1 (for odds ratios and covariate coefficients see Table S2). The predicted probability of suicidality amongst participants who perceived themselves to be overweight increased from 5.7 percentage points in 1999-2001 to 7 points in 2007-2009, 8.9 points in 2011-2013, and 10.1 points in 2015-2017. Using 1999-2001 as a baseline, our difference-in-difference analysis confirmed that participants in the perceived overweight group were disproportionately more likely to experience suicidality in 2011-2013 (an increase of 3.1 points) and
2015-2017 (increase of 4.4 points), as shown in Table 1 . Adjusting for a categorical measure of body weight or including a depression indicator did not affect the main study results (Table S3).

Our examination of individual suicidality indicators revealed that the probability of suicidal ideation associated with perceived overweight grew from 5.7 percentage points in 1999-2001 to 9.5 percentage points in 2015-2017, a statistically significant increase of 3.8 points (Table S4). The probability of making a suicide plan increased from 4.1 percentage points to 7.1 points over the same period, a significant increase of 3 points. Finally, the probability of a suicide attempt rose from 2.8 to 4.3 percentage points, a significant increase of 1.5 points.

\section{Discussion}

In a nationally representative sample of over 100,000 adolescents perceiving oneself as being overweight was associated with a 7.7 percentage point increase in self-reported suicidality from 1999 to 2017 . Over 30\% of adolescents self-identified as overweight and consistent with prior research $[3,14]$ perceived overweight predicted suicidality independent of actual BMI. Crucially, our analyses showed that the association between self-identification as overweight and suicidality grew from 5.7 percentage points in 1999-2001 to 10.1 percentage points by 2015-2017, with the greatest increases occurring after 2009. These findings indicate that perceived overweight may be a commonly experienced and increasingly important risk factor for suicidality in the teenage years.

One potential explanation for this finding is that the widespread explicit bias against heavier body weight identified in the USA has intensified $[8,9]$ which may have amplified the stigma and stress associated with identifying as overweight. In a context where the stigmatization of heavier body weight has become increasingly pervasive and socially acceptable those personally identifying as overweight may be particularly likely to internalize this stigma and to fear negative social evaluation from others [15]. These deleterious psychological effects may trigger distress, suicidal ideation, and in more extreme cases suicide plans or attempts [16].

This research had several strengths, including a large representative sample of adolescents evaluated using a consistent survey methodology over two decades. One potential limitation is that we restricted covariates to basic demographic factors (age, sex, race/ethnicity), BMI, and depression because our central purpose was to use available data to examine changes in the perceived overweight-suicidality link over time. Prior research has shown that the association between perceived overweight 
and suicidality is robust to extensive adjustment for covariates including health behavior, unhealthy weight control practices, and negative feelings [14, 17, 18]. However, it may be of interest in future to examine whether any patterns of behavior (e.g., increased adoption of maladaptive coping behaviors) can explain why the association between perceived overweight and suicidality has increased over time.

\section{Conclusions}

In a large sample of US adolescents the increased risk of suicidality associated with perceived overweight grew substantially from 2009 to 2017 . Reducing levels of weight stigma and bias may counteract the risk of suicidality associated with self-identifying as overweight.

Acknowledgements The authors are grateful to the Centers for Disease Control and Prevention, for their management of these data and for making them available at www.cdc.gov/yrbs (accessed on January 27th 2020). However, these organizations bear no responsibility for the analysis or interpretation of the data.

\section{Compliance with ethical standards}

Conflict of interest The authors declare that they have no conflict of interest.

Publisher's note Springer Nature remains neutral with regard to jurisdictional claims in published maps and institutional affiliations.

\section{References}

1. Luppino FS, de Wit LM, Bouvy PF, Stijnen T, Cuijpers P, Penninx BW, et al. Overweight, obesity, and depression: a systematic review and meta-analysis of longitudinal studies. Arch Gen Psychiatry. 2010;62:220-9.

2. Amiri S, Behnezhad S. Body mass index and risk of suicide: a systematic review and meta-analysis. $J$ Affect Disord. 2018;238:615-25.

3. Haynes A, Kersbergen I, Sutin A, Daly M, Robinson E. Does perceived overweight increase risk of depressive symptoms and suicidality beyond objective weight status? A systematic review and meta-analysis. Clin Psychol Rev. 2019;73:101753.
4. Wu YK, Berry DC. Impact of weight stigma on physiological and psychological health outcomes for overweight and obese adults: a systematic review. J Adv Nurs. 2018;74:1030-42.

5. Emmer C, Bosnjak M, Mata J. The association between weight stigma and mental health: a meta-analysis. Obes Rev. 2020;21: e12935.

6. Almond D, Lee A, Schwartz AE. Impacts of classifying New York City students as overweight. PNAS. 2016;113:3488-91.

7. Health and Social Care Information Centre. National child measurement programme-England 2012/13 school year. Leeds: Government Statistical Service; 2013.

8. Andreyeva T, Puhl RM, Brownell KD. Changes in perceived weight discrimination among Americans, 1995-1996 through 2004-2006. Obesity. 2008;16:1129-34.

9. Tomiyama AJ, Finch LE, Belsky AC, Buss J, Finley C, Schwartz MB, et al. Weight bias in 2001 versus 2013: contradictory attitudes among obesity researchers and health professionals. Obesity. 2015;23:46-53.

10. Whitehead R, Berg C, Cosma A, Gobina I, Keane E, Neville F, et al. Trends in adolescent overweight perception and its association with psychosomatic health 2002-2014: evidence from 33 countries. J Adolesc Health. 2017;60:204-11.

11. Brener ND, Kann L, Shanklin S, Kinchen S, Eaton DK, Hawkins $\mathrm{J}$, et al. Methodology of the youth risk behavior surveillance system-2013. Morb Mortal Wkly Rep. 2013;62:1-20.

12. Long JS, Freese J. Regression models for categorical dependent variables using Stata. 3rd ed. College Station, TX: Stata Press; 2014.

13. Dutton GR, Bodell LP, Smith AR, Joiner TE. Examination of the relationship between obesity and suicidal ideation. Int $\mathrm{J}$ Obes. 2013;37:1282-6.

14. Eaton DK, Lowry R, Brener ND, Galuska DA, Crosby AE. Associations of body mass index and perceived weight with suicide ideation and suicide attempts among US high school students. Arch Pediatr Adolesc Med. 2005;159:513-9.

15. Hunger JM, Major B, Blodorn A, Miller CT. Weighed down by stigma: how weight-based social identity threat contributes to weight gain and poor health. Soc Personal Psychol Compass. 2015;9:255-68.

16. Hunger JM, Dodd DR, Smith AR. Weight-based discrimination, interpersonal needs, and suicidal ideation. Stigma Health. 2019. https://doi.org/10.1037/sah0000188.

17. Swahn MH, Reynolds MR, Tice M, Miranda-Pierangeli MC, Jones CR, Jones IR. Perceived overweight, BMI, and risk for suicide attempts: findings from the 2007 Youth Risk Behavior Survey. J Adolesc Health. 2009;45:292-5.

18. Brown DR, Blanton CJ. Physical activity, sports participation, and suicidal behavior among college students. Med Sci Sports Exerc. 2002;34:1087-96. 